\title{
Characteristic Changes in Carbohydrate Profile in the Kidneys of Hereditary Nephrotic Mice (ICGN Strain)
}

\author{
Kotaro TAMURA, Noboru MANABE*, Kozue UCHIO, Makoto MIYAMOTO, Misuzu YAMAGUCHI, \\ Atsuo OGURA ${ }^{1)}$, Yoshie YAMAMOTO ${ }^{1)}$, Nobuo NAGANO ${ }^{2)}$, Yoshihiro FURUYA ${ }^{2)}$ and Hajime MIYAMOTO \\ Unit of Anatomy and Cell Biology, Department of Animal Sciences, Kyoto University, Kyoto 606-8502, ${ }^{1}$ Department of Veterinary \\ Science, National Institute of Infectious Diseases, Tokyo 162-8640, and ${ }^{2)}$ Pharmaceutical Research Laboratory, Kirin Brewery Co.,Ltd., \\ Takasaki 370-1202, Japan
}

(Received 14 September 1999/Accepted 6 December 1999)

ABSTRACT. The ICR-derived glomerulonephritis (ICGN) mice consist of heterozygous and homozygous groups and are considered to be a good model for human idiopathic nephrotic syndrome. To reveal changes in cell-surface carbohydrate construction, 24 lectins were applied to kidney sections of 10-, 30- and 50-week-old male heterozygous and homozygous ICGN mice and age-matched male ICR mice. Bandeiraea simplicifolia lectin-I (BSL-I), which specifically binds to $\alpha$-D-galactopyranosyl groups, showed positive staining in the glomeruli of ICGN mice, but not in those of ICR mice. Positive BSL-I staining was observed only in distal tubules of homozygous ICGN mice. Lectin blotting for BSL-I demonstrated characteristic glycoproteins (45, 58 and $64 \mathrm{kD})$ in ICGN but not in ICR mice, and the levels of these molecules augmented in homozygous ICGN mice with the progression of renal failure. Moreover, succinylated wheat germ agglutinin, Dolichos biflorus agglutinin, Aleuria aurantia lectin and Ulex europaeus agglutinin-I showed positive staining only in the glomeruli of homozygous ICGN mice, but not in those of heterozygous ICGN or ICR mice. The staining intensities of Ricinus communis agglutinin-I, Phaseolus vulgaris agglutinin-E and -L, Lens culinaris agglutinin and Erythrina cristagalli agglutinin (ECL) in the glomeruli of homozygous ICGN mice were stronger than those of heterozygous ICGN and ICR mice. In conclusion, lectin histochemistry provided useful information for the diagnosis and prognosis of nephrotic lesions. Characteristic BSL-I binding glycoproteins may be pathogenic factors which cause renal disease in ICGN mice and are good tools to investigate the molecular mechanism of renal disorders in ICGN mice.-KEY WORDS: cell-surface carbohydrate, glomerulonephritis, hereditary nephrotic mouse, lectin histochemistry.

J. Vet. Med. Sci. 62(4): 379-390, 2000

The ICR-derived glomerulonephritis (ICGN) mice, a novel inbred strain of mice with a hereditary nephrotic syndrome of unknown etiology, are considered to be a good model for human idiopathic nephrotic syndrome. They were established at the National Institute of Infectious Diseases [21-27]. The ICGN mice were categorized into heterozygous (approximately $40 \%$ of mice with symptoms) and homozygous (100\% of mice with severe symptoms) groups based on their outward appearance and clinical and histopathological findings. Approximately $40 \%$ of animals of each generation of ICGN mice were homozygotes, and all of the homozygous ICGN mice had severe glomerulonephritis, showing heavy proteinuria, hypoproteinemia, hypoalbuminemia and hyperlipidemia, and most developed serious anemia and severe systemic edema [21-27, 14-17, 31-33]. In homozygous ICGN mice with severe glomerulonephritis, the kidney exhibited a slightly whitish and granular surface, the renal cortex became thinner, and fibrous lesions were scattered in the renal cortex zone. In the glomeruli, thickened basement membrane consisting of multilaminar splitting, narrowed and occluded capillary lumen, no detachment of the podocytes from the

* Correspondence to: Manabe, N., Unit of Anatomy and Cell Biology, Department of Animal Sciences, Kyoto University, Kyoto 606-8502, Japan. basement membrane and podocytes with extensively fused foot processes were observed [25, 26, 31]. Such glomerular lesions were also observed in the kidneys of neonatal ICGN mice (4-6 days after birth) [27]. In the occluded renal tubules, thickened basement membranes were also seen. Thus, the ICGN mice showed albuminuria with a rapid shift of the glomerular lesion to glomerulosclerosis during the first five weeks after birth and are considered to be a suitable nephrosis model to investigate the mechanism of human idiopathic nephrotic syndrome. However, the pathological degeneration of the renal cells in ICGN mice has not been determined in detail.

Recent studies of lectin histopathology revealed that changes in cell-surface carbohydrates detected by lectin staining are good markers for histopathological diagnosis [18]. Cell-surface carbohydrates play important roles in cell recognition and interactions during cellular differentiation, and changes in cell-surface carbohydrates have been observed in various pathological conditions in both humans and experimental animals. For instance, tumor cells with malignant phenotypes show neoplastic transformation-related changes in oligosaccharide biosynthesis [2]. Cell-surface carbohydrates are reliable markers for histopathological diagnosis, and lectins recognizing these macromolecules or cell-surface carbohydrates have been extensively used as biochemical and histopathological probes in cell biology and pathology $[4,5,7,12,13]$. 
To identify the key factors in the pathogenesis and the markers for renal failure, in the present study, we examined differences in cell-surface carbohydrate structures in the kidneys of heterogenous and homogenous ICGN and normal ICR mice using 24 kinds of biotinylated lectins as histopathological probes.

\section{MATERIALS AND METHODS}

Animals and tissue preparation: Nephrotic mice (ICGN strain) were prepared by mating between homozygous males (nep/nep) and heterozygous females (nep/-) at the laboratory of National Institute of Infectious Diseases [21-27]. Ten-, 30- and 50-week-old male homozygous and heterozygous ICGN mice and age-matched male ICR mice purchased from Clea Japan (Tokyo, Japan) were used. All animals were given a standard diet (CM, Oriental Yeast Co., Tokyo, Japan) and tap water ad libitum in an air conditioned room $\left(23 \pm 1^{\circ} \mathrm{C}\right)$, under controlled lighting conditions (12L/12D). They received humane care as outlined in the "Guide for the Care and Use of Laboratory Animals" (Kyoto University Animal Care Committee according to NIH \#86-23; revised 1985). For clinical biochemical analyses, urine samples during the $24 \mathrm{hr}$ before sacrifice ( $24 \mathrm{hr}$ urine samples) were collected, and blood samples were obtained from the cervical vein under ether anesthesia. Serum was prepared by centrifugation at $1,000 \mathrm{rpm}$ for $10 \mathrm{~min}$. The animals were killed under deep ether anesthesia, and then the kidneys were rapidly removed. The left kidney was immediately fixed in $10 \%$ neutral-buffered formalin $(\mathrm{pH}$ 7.4) for conventional histopathological evaluation and lectin histochemistry, while the right kidney was frozen in liquid nitrogen for histochemical measurement of total and type I collagens and for lectin blot analysis.

Clinical biochemical analysis: To evaluate nephrotic state, $24 \mathrm{hr}$ urine and serum samples were examined on the basis of the following biochemical parameters [32]. Urinary and serum albumin (uAlb and sAlb, respectively) levels were measured by a bromo-cresol green method using an A/GB test kit (Wako Pure Chemical Co., Osaka, Japan) to assess the severity of albuminuria and hypoalbuminemia. To assess the loss of renal function, serum creatinine $(\mathrm{sCr})$ and blood urea nitrogen (BUN) levels were measured by Jaffé's method with a creatinine test kit (Wako) and by the urease indophenol method with a nitrogen-B test kit (Wako), respectively. To evaluate the degree of hypercholesteremia, the serum total cholesterol (sTC) level was determined by an enzymatic method with a Cholesterol-E test kit (Wako). All procedures were performed according to the manufacturers' protocols.

Renal histopathology: After fixation, the kidney samples were dehydrated through a graded ethanol series and embedded in Histosec (Merck Co., Darmstadt, Germany). Sections $3 \mu \mathrm{m}$ thick were prepared on a microtome, mounted on glass slides precoated with 3-aminopropyltriethoxysilane (Aldrich Chemical, Milwaukee, WI, U.S.A.), deparaffinized with xylene and rehydrated through a graded ethanol series.
For conventional histopathological evaluation, some of the sections were stained with hematoxylin and eosin according to the standard method. As previously reported [10, 32, 33], the extent of glomerulosclerosis was expressed as the degree of collagen deposition, which was assessed on sections stained with Sirius red solution (saturated picric acid in distilled water containing $0.1 \%$ Sirius red F3B; BDH Chemicals Ltd., Poole, UK) [8-10]. All slides were mounted with Entellan (Merck), and examined by light microscopy at least three sections/mouse. In each kidney specimen, approximately 100 glomeruli were selected at random and evaluated by light microscopy as described previously [10, 32, 33]. Briefly, the mesangial expansion in the glomeruli was scored according to the extent of the sclerotic lesion in the glomerulus and graded from 0 to $4(0$ $=$ normal $; 1=$ change affecting $<25 \% ; 2=$ change affecting $<25-50 \% ; 3=$ change affecting $<50-75 \% ; 4=$ change affecting $>75 \%$ ). In addition, the morphological changes in the glomeruli (capillary aneurysm and hypercellularity) [29], and tubular (cystic tubular dilation, epithelial cellular atrophy and intraluminal cast formation) [30] and tubulointerstitial (tubulointerstitial expansion and mononuclear cell filtration around arterioles) [20] lesions were also recorded. Each glomerulus was classified into three categories: normal (glomerulus without mesangial expansion), expansion of mesangial areas (glomerulus graded from 1 to 4 ), and capillary aneurysm (glomerulus with severe degeneration, i.e. not only with expansion of mesangial but also with capillary aneurysm and hypercellularity). Morphological changes of glomeruli were expressed as percentage of glomeruli in each category.

Histochemical quantification of total and type I collagens: The degree of ECM deposition in kidney sections is a good indicator of glomerular sclerosis. Total and type I collagen levels in each frozen section of mouse kidney cortex were measured by microquantitation method as previously described [8-10].

Lectin histochemistry and blot analysis: To identify the localization of specific carbohydrate chains, paraffinembedded serial sections were incubated with 24 biotinylated lectins. Carbohydrate binding specificities of the 24 lectins are summarized in Table 1. The localization of specific carbohydrate chains in the kidney sections was visualized using the avidin-biotin peroxidase complex (ABC) method as previously described [4, 5, 12, 13]. Briefly, after deparaffinization and rehydration, the sections were incubated with avidin and biotin blocking solution (Vector Laboratories, Burlingame, CA, U.S.A.) at room temperature (RT; $23-25^{\circ} \mathrm{C}$ ) for 15 min to block endogenous binding sites, and then washed with PBS. The sections were incubated with biotinylated lectins diluted appropriately with PBS (see Table 1) for $24 \mathrm{hr}$ at $4{ }^{\circ} \mathrm{C}$, washed with $\mathrm{PBS}$, and then incubated with $\mathrm{ABC}$ reagent (Vector) for $60 \mathrm{~min}$ at RT. After washing, they were colorized with $0.002 \% \mathrm{H}_{2} \mathrm{O}_{2}, 0.1 \%$ 3,3'-diaminobenzidine$4 \mathrm{HCl}$ (DAB; Wako) in $0.1 \mathrm{M}$ phosphate buffer ( $\mathrm{pH} 7.4)$, and then counterstained with Mayer's hematoxylin. After 
Table 1. Carbohydrate binding specificities of the lectins used

\begin{tabular}{|c|c|c|c|}
\hline Lectin & Source & $\begin{array}{c}\left.\text { Dilution }^{\mathrm{c}}\right) \\
(\mu \mathrm{g} / \mathrm{m} l)\end{array}$ & Binding specificity ${ }^{\mathrm{d})}$ \\
\hline Jacalina $^{a)}$ & Artocarpus integrifolia & 4 & $\alpha$-Gal \\
\hline $\mathrm{LEL}^{\mathrm{a})}$ & Lycopersicon esculentum & 2 & $\beta$-GlcNAc, Poly LacNAc, GlcNAc oligomer \\
\hline RCA-Ia) & Ricinus communis & 4 & $\beta$-GalNAc, $\beta$-Gal, Gal $\beta 1 \rightarrow$ GlcNAc on complex glycan \\
\hline $\mathrm{SSA}^{\mathrm{b})}$ & Sambucus sieboldiana & 3 & $\mathrm{Sia} \alpha 2 \rightarrow 6 \mathrm{Gal} / \mathrm{GalNAc}$ \\
\hline WGA $^{\text {a) }}$ & Triticum vulgaris & 3 & $\beta$-GlcNAc, Poly LacNAc, GlcNAc oligomer, Sia \\
\hline $\mathrm{DSL}^{\mathrm{a})}$ & Datura Stramonium & 2 & $\beta$-GlcNAc, Poly LacNAc, Tri/tetra-antenna complex glycan \\
\hline $\mathrm{STL}^{\mathrm{a})}$ & Solanum tuberosum & 4 & $\beta$-GlcNAc, Poly LacNAc, GlcNAc oligomer \\
\hline $\mathrm{AAL}^{\mathrm{b})}$ & Aleuria aurantia & 2 & Fuc $\alpha 1 \rightarrow 6 \mathrm{GlcNAc}$ \\
\hline MAM $^{\mathrm{b})}$ & Maackia amurensis & 3 & $\mathrm{Sia} \alpha 2 \rightarrow 3 \mathrm{Gal}$ \\
\hline PHA-Ea) & Phaseolus vulgaris & 2 & Bisected complex glycan \\
\hline PHA-La) & Phaseolus vulgaris & 2 & Tri/tetra-antenna complex glycan \\
\hline $\mathrm{LCA}^{\mathrm{a})}$ & Lens culinaris & 3 & $\alpha$-Man, complex glycan with fucosylated core \\
\hline $\mathrm{PSA}^{\mathrm{a})}$ & Pisum sativum & 3 & $\alpha$-Man, complex glycan with fucosylated core \\
\hline $\mathrm{SJA}^{\mathrm{a})}$ & Sophora japonica & 8 & $\beta$-GalNAc \\
\hline $\mathrm{DBA}^{\mathrm{a})}$ & Dolichos biflorus & 8 & $\alpha$-GalNAc, GalNAc $\alpha 1 \rightarrow 3$ GalNAc (Gal) \\
\hline ECLa) & Erythrina cristagalli & 4 & $\beta$-Gal, $\beta$-GalNAc \\
\hline PNAa) & Arachis hypogaea & 8 & $\beta$-Gal, O-linked glycan, Gal $\beta 1 \rightarrow 3$ GalNAc \\
\hline $\mathrm{SBA}^{\mathrm{a})}$ & Glycine $\max$ & 8 & $\alpha$-GalNAc, O-linked glycan, GalNAc $\alpha 1 \rightarrow 3 \mathrm{Gal}$ \\
\hline UEA-Ia) & Ulex europaeus & 8 & $\alpha$-Fuc, Fuc $\alpha 1 \rightarrow 2 \mathrm{Gal}$ \\
\hline BSL-II $\left.{ }^{a}\right)$ & Bandeiraea simplicifolia & 6 & $\alpha-, \beta$-GlcNAc \\
\hline Con $\mathrm{A}^{\mathrm{a})}$ & Canavalia ensiformis & 4 & $\alpha$-Man, oligomannoside glycan, Bi-antenna complex glycan \\
\hline BSL-Ia) & Bandeiraea simplicifolia & 6 & $\alpha$-GalNAc, $\alpha$-Gal, Gal $1 \rightarrow 3 \mathrm{Gal}$ \\
\hline $\mathrm{VVA}^{\mathrm{a})}$ & Vicia villosa & 6 & $\beta$-GalNAc, O-linked glycan, GalNAc $\alpha 1 \rightarrow 3 \mathrm{Gal}$ \\
\hline s-WGA ${ }^{a}$ & Triticum vulgaris & 8 & $\beta$-GlcNAc, poly LacNAc, GlcNAc oligomer \\
\hline
\end{tabular}

a) Biotinylated lectins were purchased from Vector Laboratories (Burlingame, CA, U.S.A.).

b) Biotinylated lectins were purchased from Seikagaku Kogyo Co. (Tokyo, Japan).

c) Biotinylated lectins were diluted with PBS.

d) Abbreviation: Fuc: fucose, Gal: D-galactose, GalNAc: N-acetylgalactosamine, GlcNAc: N-acetylglucosamine, LacNAc: N-acetyllactosamine, Man: mannose, Sia: sialic acid.

dehydration, they were mounted in Entellan and examined under a light microscope. Negative control sections were incubated without biotinylated lectins or $\mathrm{ABC}$ reagent. As specific controls, lectins were incubated with $0.002-0.2 \mathrm{M}$ solutions of corresponding sugar haptens (see Table 1) before and during application of the lectin solution to the tissue sections [3-6, 12, 13, 19, 34].

As characteristic staining of Bandeiraea simplicifolia lectin-I (BSL-I) was demonstrated histochemically, lectin blot analysis was performed as previously described $[4,5$, 12]. Briefly, frozen kidney sections (at least $10 \mathrm{mg}$ ) were mixed with $0.0625 \mathrm{M}$ Tris- $\mathrm{HCl}(\mathrm{pH} 6.8)$ containing $2 \%$ Nonidet-P40, 2\% sodium dodecyl sulfate (SDS) and 5\% 2mercaptoethanol (Sigma Chemical, St. Louis, MO, U.S.A.), heated for $3 \mathrm{~min}$ at $100^{\circ} \mathrm{C}$, and then urea was added at a final concentration of $8 \mathrm{M}$. The protein concentration of each sample was determined by a modification of the method of Bradford [1]. The sample proteins (30 $\mu \mathrm{g} / \mathrm{lane})$ and molecular weight standard (Sigma) were fractionated by $7.5 \%$ SDS-polyacrylamide gel electrophoresis (SDSPAGE). The fractionated proteins were visualized by staining with Coomassie brilliant blue (CBB), and then subsequently transferred electrophoretically onto polyvinylidene difluoride (PVDF) membranes (Millipore, Bedford, MA, U.S.A.). The PVDF membranes were preincubated with $10 \mathrm{mM}$ Tris- $\mathrm{HCl}$ buffer $(\mathrm{pH} 7.4)$ containing $500 \mathrm{mM} \mathrm{NaCl}, 0.05 \%$ Tween 20 (TST), and then incubated with biotinylated BSL-I. After washing with TST, they were reacted with HRP-conjugated avidin (Honen Corporation, Tokyo, Japan), washed, and then colorized with $100 \mathrm{mM}$ phosphate buffer ( $\mathrm{pH} 7.4$ ) containing $0.003 \% \mathrm{H}_{2} \mathrm{O}_{2}$ and $0.03 \% \mathrm{DAB}$.

Statistical analysis: ANOVA analysis with Fisher's least significant differences test comparison for biochemical data, and Wilcoxon's signed rank test for morphologic estimation were carried out with the Statview IV program using a Macintosh computer. Differences at a probability of $p<0.05$ were considered significant. All data are expressed as mean values $\pm \mathrm{SD}(\mathrm{n}=10)$.

\section{RESULTS}

Clinical parameters: Aged homozygous ICGN mice (50 weeks) manifested clinical abnormalities such as the exercise intolerance, pale ears, weight loss as well as edema. Urinary and serum biochemical data are summarized in Table 2. There were no significant differences in serum albumin, creatinine, total cholesterol or BUN between ICR and heterozygous ICGN mice at any time point examined, but low levels of urine albumin (weak albuminuria) and mild disorders in other nephrotic parameters were seen in heterozygous ICGN mice. The nephrotic state progressed 
Table 2. Urine and serum biochemical features in control (ICR), heterozygous and homozygous ICGN mice

\begin{tabular}{|c|c|c|c|c|c|c|c|c|c|}
\hline \multirow{2}{*}{$\begin{array}{l}\text { Strains } \\
\text { Age (weeks old) }\end{array}$} & \multicolumn{3}{|c|}{ ICR } & \multicolumn{3}{|c|}{$\begin{array}{l}\text { Heterozygous } \\
\text { ICGN } \\
\end{array}$} & \multicolumn{3}{|c|}{$\begin{array}{l}\text { Homozygous } \\
\text { ICGN } \\
\end{array}$} \\
\hline & 10 & 30 & 50 & 10 & 30 & 50 & 10 & 30 & 50 \\
\hline $\begin{array}{l}\text { Urine albumin } \\
(\mathrm{mg} / \mathrm{m} l)\end{array}$ & ND & ND & ND & ND & $1.4 \pm 0.8$ & $2.3 \pm 1.9$ & $8.8 \pm 1.5^{* *}$ & $8.8 \pm 1.5^{* *} *$ & $21.4 \pm 3.8^{* *}$ \\
\hline $\begin{array}{l}\text { Serum albumin } \\
(\mathrm{g} / \mathrm{d} l)\end{array}$ & $3.10 \pm 0.08$ & $3.14 \pm 0.11$ & $3.11 \pm 0.13$ & $2.99 \pm 0.07$ & $3.08 \pm 0.15$ & $2.98 \pm 0.11$ & $2.55 \pm 0.25$ & $2.01 \pm 0.33^{*}$ & $1.97 \pm 0.31 *$ \\
\hline $\begin{array}{l}\text { Serum creatinine } \\
(\mathrm{mg} / \mathrm{d} l)\end{array}$ & $0.31 \pm 0.06$ & $0.31 \pm 0.09$ & $0.28 \pm 0.07$ & $0.33 \pm 0.07$ & $0.37 \pm 0.11$ & $0.41 \pm 0.10$ & $0.39 \pm 0.13$ & $0.89 \pm 0.15 * *$ & $1.24 \pm 0.24 * *$ \\
\hline $\begin{array}{l}\text { Blood urea nitrogen } \\
(\mathrm{mg} / \mathrm{d} l)\end{array}$ & $29.8 \pm 4.1$ & $32.2 \pm 3.1$ & $29.5 \pm 3.3$ & $33.1 \pm 3.1$ & $36.8 \pm 7.1$ & $39.2 \pm 8.4$ & $43.1 \pm 7.4$ & $67.5 \pm 11.4^{*}$ & $87.3 \pm 20.7 * *$ \\
\hline $\begin{array}{l}\text { Serum total cholesterol } \\
(\mathrm{mg} / \mathrm{d} l)\end{array}$ & $95.1 \pm 8.5$ & $119.6 \pm 9.7$ & $145.4 \pm 15.8$ & $104.1 \pm 8.2$ & $136.9 \pm 18.2$ & $156.9 \pm 20.5$ & $140.4 \pm 8.8$ & $205.1 \pm 30.5^{*}$ & $285.6 \pm 30.5 * *$ \\
\hline
\end{tabular}

For details, see Materials and Methods.

ND: Not detected.

$*$ and $* *: p<0.05$ and 0.01 vs each heterozygous ICGN group.

Table 3. Total and type I collagen levels in the renal cortex of control (ICR), heterozygous and homozygous ICGN mice

\begin{tabular}{|c|c|c|c|c|c|c|c|c|c|}
\hline \multirow{2}{*}{$\begin{array}{l}\text { Strains } \\
\text { Age (weeks old) }\end{array}$} & \multicolumn{3}{|c|}{ ICR } & \multicolumn{3}{|c|}{$\begin{array}{l}\text { Heterozygous } \\
\text { ICGN }\end{array}$} & \multicolumn{3}{|c|}{$\begin{array}{l}\text { Homozygous } \\
\text { ICGN }\end{array}$} \\
\hline & 10 & 30 & 50 & 10 & 30 & 50 & 10 & 30 & 50 \\
\hline $\begin{array}{l}\text { Total collagen } \\
\text { (mg/g protein) } \\
\text { Type I collagen }\end{array}$ & $4.49 \pm 0.59$ & $4.88 \pm 0.73$ & $4.63 \pm 0.80$ & $4.86 \pm 0.91$ & $5.22 \pm 1.21$ & $5.75 \pm 1.39$ & $5.02 \pm 1.84$ & $7.03 \pm 2.42^{*}$ & $9.47 \pm 2.87 * *$ \\
\hline (mg/g protein) & $1.78 \pm 0.28$ & $1.93 \pm 0.33$ & $1.84 \pm 0.38$ & $1.94 \pm 0.31$ & $2.02 \pm 0.42$ & $2.45 \pm 0.56$ & $2.02 \pm 0.31$ & $3.02 \pm 0.55^{*}$ & $4.06 \pm 0.62 * *$ \\
\hline
\end{tabular}

For details, see Materials and Methods.

$*$ and **: $p<0.05$ and 0.01 vs each heterozygous ICGN group.

in an age-dependent manner in homozygous ICGN mice. Thirty- and 50-week-old homozygous ICGN mice showed severe albuminuria (6.29-fold and 9.30-fold increases as compared with age-matched heterozygous ICGN mice) and hypoalbuminemia (1.53-fold and 1.51-fold decreases as compared with age-matched heterozygous ICGN mice). Marked increases in $\mathrm{sCr}$ (2.41-fold and 3.02-fold increases as compared with age-matched heterozygous ICGN mice) and BUN (1.83-fold and 2.23-fold increase as compared with age-matched heterozygous ICGN mice) indicated the loss of renal function in 30- and 50-week-old homozygous ICGN mice. Increased serum total cholesterol (1.50-fold and 1.82-fold increases as compared with age-matched heterozygous ICGN mice) suggested severe hypercholesterolemia in 30- and 50-week-old homozygous ICGN mice.

Renal collagen levels and histopathology: Renal total and type I collagen levels assessed by a microquantification method are shown in Table 3 . There were no significant differences in total or type I collagen levels in kidney cortex sections between ICR and heterozygous ICGN mice at any time point examined. When compared with 30- and 50week-old heterozygous ICGN mice, age-matched homozygous ICGN mice showed 1.35-fold and 1.65-fold increases in total collagen levels, and 1.49-fold and 1.66fold increases in type I collagen levels.
Histopathological examination revealed that most ICR and heterozygous ICGN mice had kidneys with normal glomeruli (more than $98 \%$ and $89 \%$, respectively), but all 50-week-old homozygous ICGN mice had kidneys with abnormal glomeruli $(77 \pm 9 \%$ and $23 \pm 9 \%$ of glomeruli with expanded mesangial area and with capillary aneurysm, respectively; see Table 4). There were no marked differences in renal histopathological findings between ICR (used as normal healthy controls) and heterozygous ICGN mice (Fig. 1A and C). However, expansion of the mesangial areas with an apparent increase in the mesangial matrix, appearance of cysts, extension of renal tubules and infiltration of inflammatory cells were observed in the kidney sections of 50-week-old homozygous ICGN mice (Fig. 1B and D). Such histopathological abnormalities progressed in an age-dependent manner in homozygous ICGN mice. In aged heterozygous ICGN mice, kidneys exhibited moderate expansion of mesangial matrix $(9 \pm 2 \%$ and $2 \pm 1 \%$ of glomeruli with expansion of mesangial areas and with capillary aneurysm, respectively; see Table 4), but no proliferation of mesangial cells was observed. Moreover, when compared with 50-week-old ICR and heterozygous ICGN mice (Fig. 1C), progressed fibrotic degeneration in the glomeruli was observed in the kidney sections of 50week-old homozygous ICGN mice (Fig. 1D). Thus, glomerulonephritic and glomerular-fibrotic degeneration 

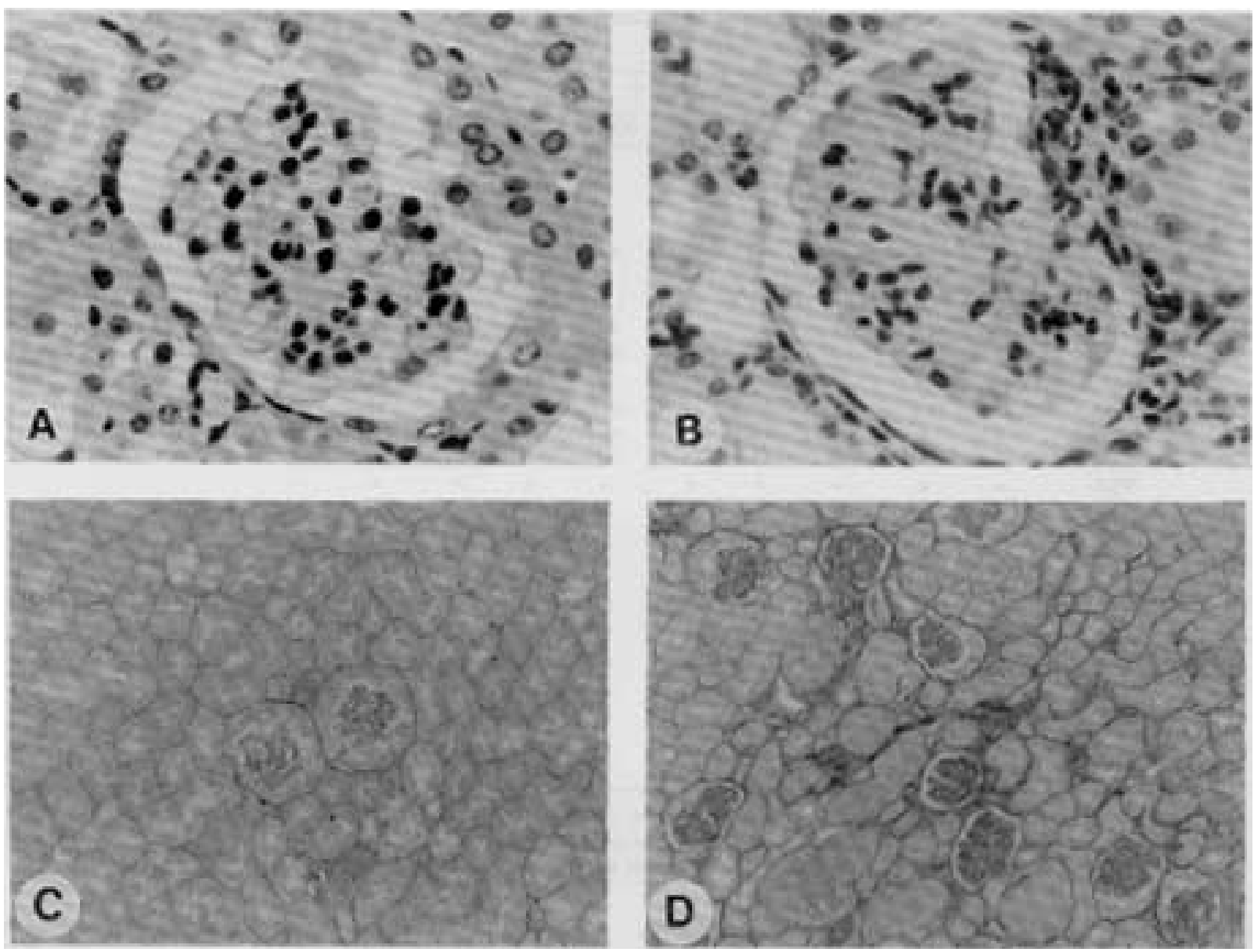

Fig. 1. Kidney sections of 50-week-old male heterozygous (A and C) and homozygous ICGN mice (B and D) were stained with hematoxylin and eosin (A and B; $\times 600)$ and with Sirius red $(\mathrm{C}$ and $\mathrm{D} ; \times 150)$. As compared with heterozygous ICGN mice (A), expansion of mesangial matrix, but no proliferation of mesangial cells was seen in the kidneys of homozygous ICGN mice (B). Severe fibrotic degeneration in glomeruli and basement membrane of renal tubules, appearance of cysts, and extension of renal tubules were observed only in the kidneys of homozygous ICGN mice (D) but not in those of heterozygous ICGN mice (C).

Table 4. Morphological changes of glomeruli in control (ICR), heterozygous and homozygous ICGN mice

\begin{tabular}{|c|c|c|c|c|c|c|c|c|c|}
\hline \multirow{2}{*}{$\begin{array}{l}\text { Strains } \\
\text { Age (weeks old) }\end{array}$} & \multicolumn{3}{|c|}{ ICR } & \multicolumn{3}{|c|}{$\begin{array}{c}\text { Heterozygous } \\
\text { ICGN }\end{array}$} & \multicolumn{3}{|c|}{$\begin{array}{l}\text { Homozygous } \\
\text { ICGN }\end{array}$} \\
\hline & 10 & 30 & 50 & 10 & 30 & 50 & 10 & 30 & 50 \\
\hline Normal $(\%)$ & $100 \pm 1$ & $98 \pm 1$ & $98 \pm 1$ & $99 \pm 1$ & $94 \pm 2$ & $89 \pm 4$ & $92 \pm 4$ & $31 \pm 11 * *$ & NO \\
\hline Expansion of mesangial areas (\%) & NO & $2 \pm 1$ & $2 \pm 1$ & $1 \pm 1$ & $5 \pm 1$ & $9 \pm 2$ & $7 \pm 2$ & $54 \pm 15^{* *}$ & $77 \pm 9 * *$ \\
\hline Capillary aneurysm (\%) & NO & NO & NO & NO & $1 \pm 1$ & $2 \pm 1$ & $1 \pm 1$ & $15 \pm 4 * *$ & $23 \pm 9 * *$ \\
\hline
\end{tabular}

These morphological changes are described in detail in Materials and Methods.

Not observed.

$* *: p<0.05$ and 0.01 vs each heterozygous ICGN group.

became severe only in homozygous ICGN mice.

Lectin histochemistry: All controls in which staining was performed following treatment with specific inhibitory sugars were negative, indicating that the lectin staining patterns observed in this study were specific. Histochemical staining intensity of each lectin in the glomerulus, proximal tubule, distal tubule and vessel walls located among the renal tubules was estimated semiquantitatively, and representative histological findings of lectin histochemistry in the kidney sections of 50-week-old ICR, heterozygous and homozygous ICGN mice are summarized in Table 5. No positive staining with Sophora japonica agglutinin (SJA) was seen in any kidney sections. No differences in staining patterns of the eleven lectins, i.e. Jacalin, Lycopersicon 
Table 5. Histochemical staining patterns of biotinylated lectins in the kidney sections of 50-week-old ICR, heterozygous ICGN and homozygous ICGN mice

\begin{tabular}{|c|c|c|c|c|c|c|c|c|c|c|c|c|}
\hline & \multicolumn{3}{|c|}{ Glomerulus } & \multicolumn{3}{|c|}{ Proximal T. } & \multicolumn{3}{|c|}{ Distal T. } & \multicolumn{3}{|c|}{ Vessel wall } \\
\hline & ICR & ICGN $^{1}$ & $\overline{\text { ICGN }^{2}}$ & ICR & ICGN $^{1}$ & $\overline{\text { ICGN }^{2}}$ & $\overline{I C R}$ & ICGN $^{1}$ & $\overline{\mathrm{ICGN}^{2}}$ & $\overline{\mathrm{ICR}}$ & ICGN $^{1}$ & $\mathrm{ICGN}^{2}$ \\
\hline Jacalin & ++ & ++ & ++ & + & + & + & + & + & + & + & + & + \\
\hline LEL & ++ & ++ & ++ & + & + & + & +++ & +++ & +++ & + & + & + \\
\hline RCA-I & + & + & ++ & ++ & ++ & ++ & ++ & ++ & ++ & - & - & - \\
\hline SSA & ++ & ++ & ++ & - & - & ++ & +++ & +++ & +++ & + & + & + \\
\hline WGA & +++ & +++ & +++ & ++ & ++ & ++ & +++ & +++ & +++ & + & + & + \\
\hline DSL & + & + & + & ++ & ++ & +++ & +++ & +++ & +++ & - & - & - \\
\hline STL & ++ & ++ & ++ & ++ & ++ & ++ & +++ & +++ & +++ & + & + & + \\
\hline AAL & - & - & + & ++ & ++ & +++ & + & + & + & - & - & - \\
\hline MAM & ++ & ++ & ++ & - & - & - & +++ & +++ & +++ & + & + & + \\
\hline PHA-E & ++ & ++ & +++ & ++ & ++ & +++ & ++ & ++ & ++ & + & + & + \\
\hline PHA-L & + & + & ++ & ++ & ++ & +++ & + & + & + & - & - & - \\
\hline LCA & + & + & ++ & ++ & ++ & ++ & - & - & - & - & - & - \\
\hline PSA & + & + & + & + & + & + & + & + & + & - & - & - \\
\hline SJA & - & - & - & - & - & - & - & - & - & - & - & - \\
\hline DBA & - & - & ++ & - & - & - & +++ & +++ & +++ & - & - & +++ \\
\hline ECL & + & + & ++ & + & + & + & ++ & ++ & ++ & - & - & - \\
\hline PNA & + & + & + & + & + & + & +++ & +++ & +++ & - & - & - \\
\hline SBA & + & + & + & + & + & + & ++ & ++ & ++ & - & - & - \\
\hline UEA-I & - & - & ++ & - & - & - & - & - & - & - & - & - \\
\hline BSL-II & - & - & - & + & + & + & + & + & + & - & - & - \\
\hline ConA & + & + & + & ++ & ++ & ++ & + & + & + & - & - & - \\
\hline BSL-I & - & ++ & +++ & - & - & - & - & - & ++ & - & ++ & +++ \\
\hline VVA & + & + & + & + & + & + & +++ & +++ & +++ & - & - & - \\
\hline s-WGA & - & - & ++ & - & ++ & ++ & +++ & +++ & +++ & - & - & - \\
\hline
\end{tabular}

Abbreviations used: ICGN $^{1}$, heterozygous ICGN mice; ICGN ${ }^{2}$, homozygous ICGN mice; Proximal T., proximal tubule; Distal T., distal tubule; Vessel wall, vessel walls located between the renal tubules.

Intensity:,,,-++++++ denote negative, weak, moderate and intense staining, respectively.

esculentum lectin (LEL), wheat germ agglutinin (WGA), Solanum tuberosum lectin (STL), Maackia amurensis lectin (MAM), Pisum sativum agglutinin (PSA), peanut agglutinin (PNA), soybean agglutinin (SBA), BSL-II, concanavalin A (ConA) and Vicia villosa agglutinin (VVA), were observed in any parts of the kidney sections among ICR, heterozygous and homozygous ICGN mice. The intensity of Ricinus communis agglutinin-I (RCA-I), Phaseolus vulgaris agglutinin-E and -L (PHA-E and -L, respectively), Lens culinaris agglutinin (LCA) and Erythrina cristagalli agglutinin (ECL) staining in glomerular mesangium of homozygous ICGN mice increased with nephritis aggravation, but no such changes in lectin staining was seen in ICR or heterozygous ICGN mice. It was remarkable that no positive staining with BSL-I was seen in the kidney sections of ICR mice (Fig. 2A and B), but strong staining with BSL-I was demonstrated in glomerular capillary walls, i.e. endothelial cells of glomerular capillaries, and some their walls located among the renal tubules in the kidney sections of both heterozygous (Fig. 2C and D) and homozygous (Fig. 2E and F) ICGN mice. Luminal apical cell membranes of distal tubules were consistently stained with succinylated wheat germ agglutinin (s-WGA) in all ICR (Fig. 3A), heterozygous (Fig. 3B) and homozygous ICGN mouse kidney sections. A granular staining pattern with s-WGA was seen in epithelia of proximal tubules of a proportion of heterozygous ICGN mice and all homozygous
ICGN mice, but those in ICR mice were never stained with s-WGA. No positive staining was observed with s-WGA, Dolichos biflorus agglutinin (DBA), Aleuria aurantia lectin (AAL) or Ulex europaeus agglutinin-I (UEA-I) in glomerular mesangium in the kidney sections of ICR (Fig. 3A; s-WGA) or heterozygous ICGN mice (Fig. 3B and C; s-WGA and DBA, respectively), but positive staining with these lectins was seen in the glomerular mesangium in the sections of homozygous ICGN mice (Fig. 3D; DBA staining). In the kidneys of homozygous ICGN mice, the vessel walls located between the renal tubules as well as their interstitium were stained with DBA (Fig. 3D), but no such staining pattern with DBA was seen in the kidneys of ICR (Fig. 3C) or heterozygous-ICGN mice. No positive staining with Sambucus sieboldiana agglutinin (SSA) was seen in brush borders of extended proximal tubules of ICR or heterozygous ICGN (Fig. 3E) mice, but those of homozygous ICGN mice (Fig. 3F) were strongly stained with SSA. In brush borders of proximal tubules, staining intensity of Aleuria aurantia lectin (AAL), PHA-E and PHA-L increased with nephritis aggravation in homozygous ICGN mice. The interstitia of both proximal and distal tubules were stained with Lycopersicon esculentum lectin (LEL), SSA and Pisum sativum agglutinin (PSA), but no staining with these lectins was seen in the kidneys of ICR or heterozygous ICGN mice.

Lectin blot analysis: As described above, positive staining 

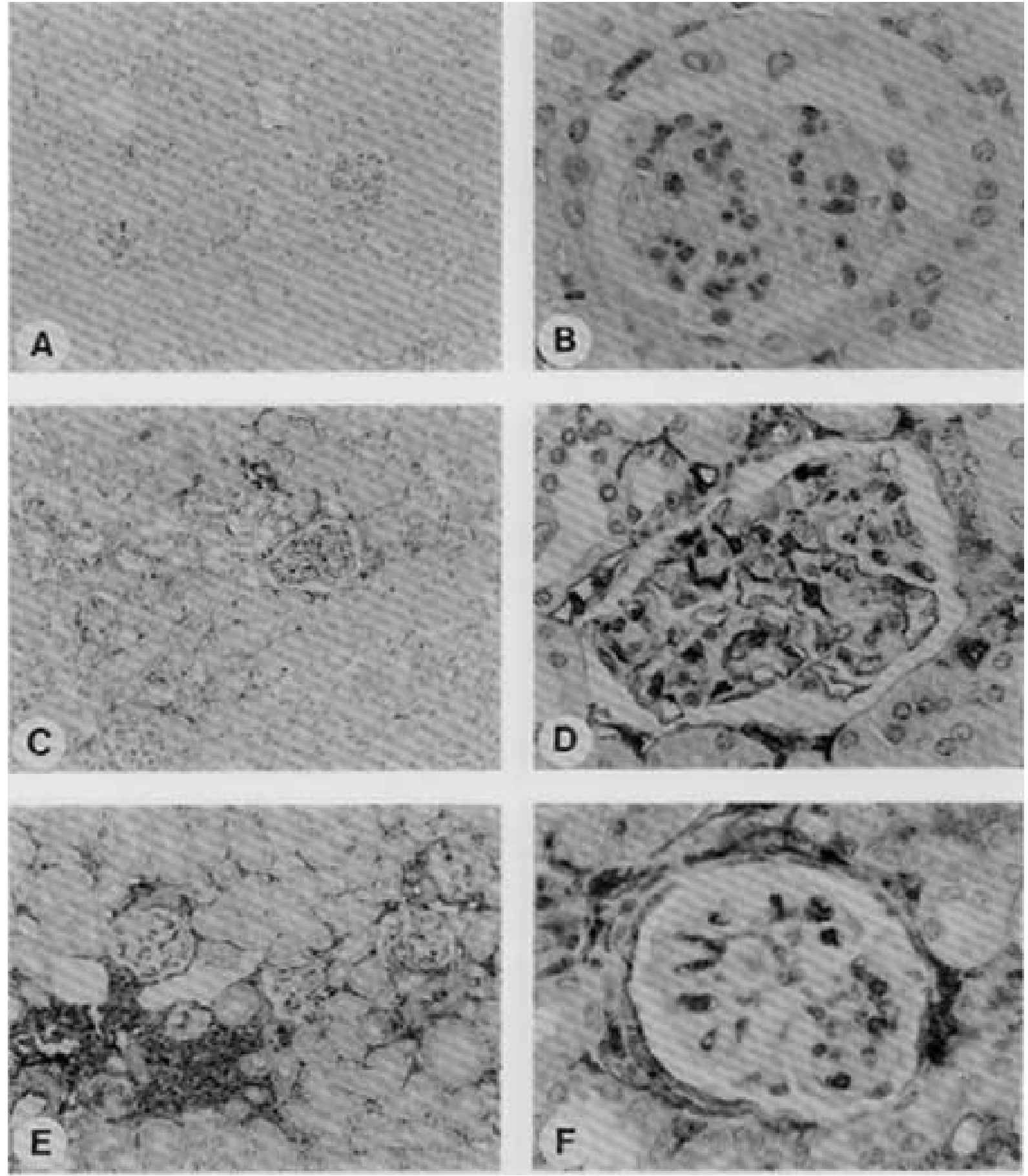

Fig. 2. Staining intensity in kidney sections of 50-week-old male ICR (A and B; $\times 150$ and 600, respectively), heterozygous (C and $\mathrm{D} ; \times 150$ and 600 , respectively) and homozygous (E and $\mathrm{F} ; \times 150$ and 600 , respectively) ICGN mice stained with Bandeiraea simplicifolia lectin-I (BSL-I). No positive staining was seen with BSL-I in the kidneys of ICR mice (A and B). In the kidneys of heterozygous (C and D) and homozygous (E and D) ICGN mice, endothelial cells of glomerular capillary vessels and some vessel walls located among the renal tubules were strongly stained with BSL-I.

with BSL-I was histochemically demonstrated in heterozygous and homozygous ICGN mice, but not in ICR mice. So, we performed lectin blot analysis with BSL-I to determine the specific glycoproteins expressed in heterozygous and homozygous ICGN mice. No distinct differences were observed in the protein bands prepared 

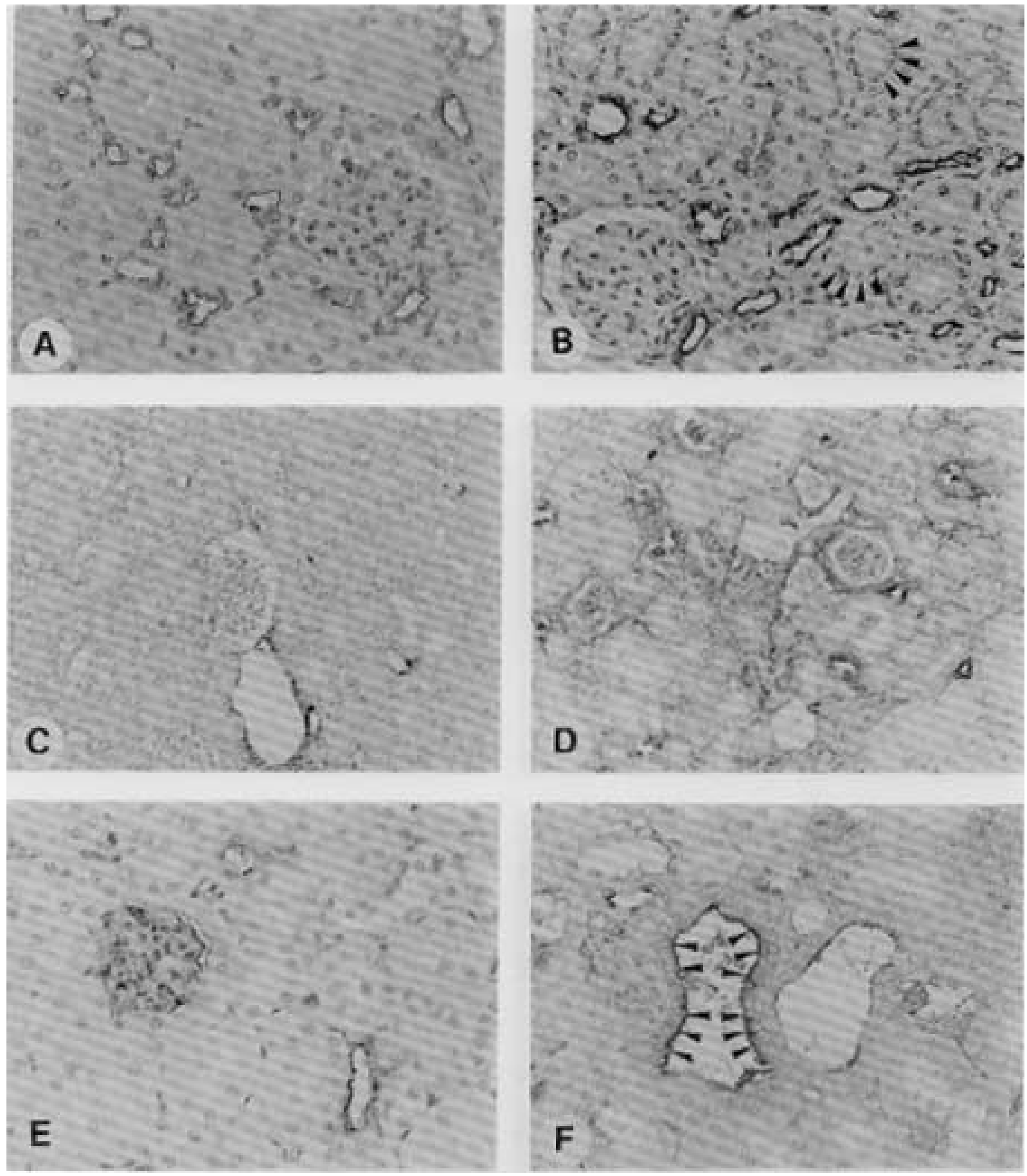

Fig. 3. Kidney sections of 50-week-old male ICR $(\mathrm{A} ; \times 300)$, heterozygous (B, C and E; $\times 300,150$ and 300 , respectively) and homozygous (D and F; $\times 150$ and 300, respectively) ICGN mice were stained with succinylated wheat germ agglutinin (s-WGA) (A and B), Dolichos biflorus agglutinin (DBA) (C and D) and Sambucus sieboldiana agglutinin (SSA) (E and F). Luminal apical cell membranes of distal tubules in the kidney sections of ICR and heterozygous ICGN mice were consistently stained with s-WGA (A and B, respectively). Granular substances stained with s-WGA (arrowheads) were seen in the epithelia of proximal tubules of heterozygous ICGN mice (B), but no such positive staining was seen in those of ICR mice (A). Positive staining with DBA in glomerular mesangium and the vessel walls located among the renal tubules as well as their interstitium was observed in the kidneys of homozygous ICGN mice (D), but no such positive staining was observed in the kidney of heterozygous ICGN mouse (C). Brush borders of extended proximal tubules were strongly stained with SSA in the kidneys of homozygous ICGN mice (F; arrowheads), but no such positive staining with SSA was seen in the kidneys of ICR or heterozygous ICGN mice (E). 


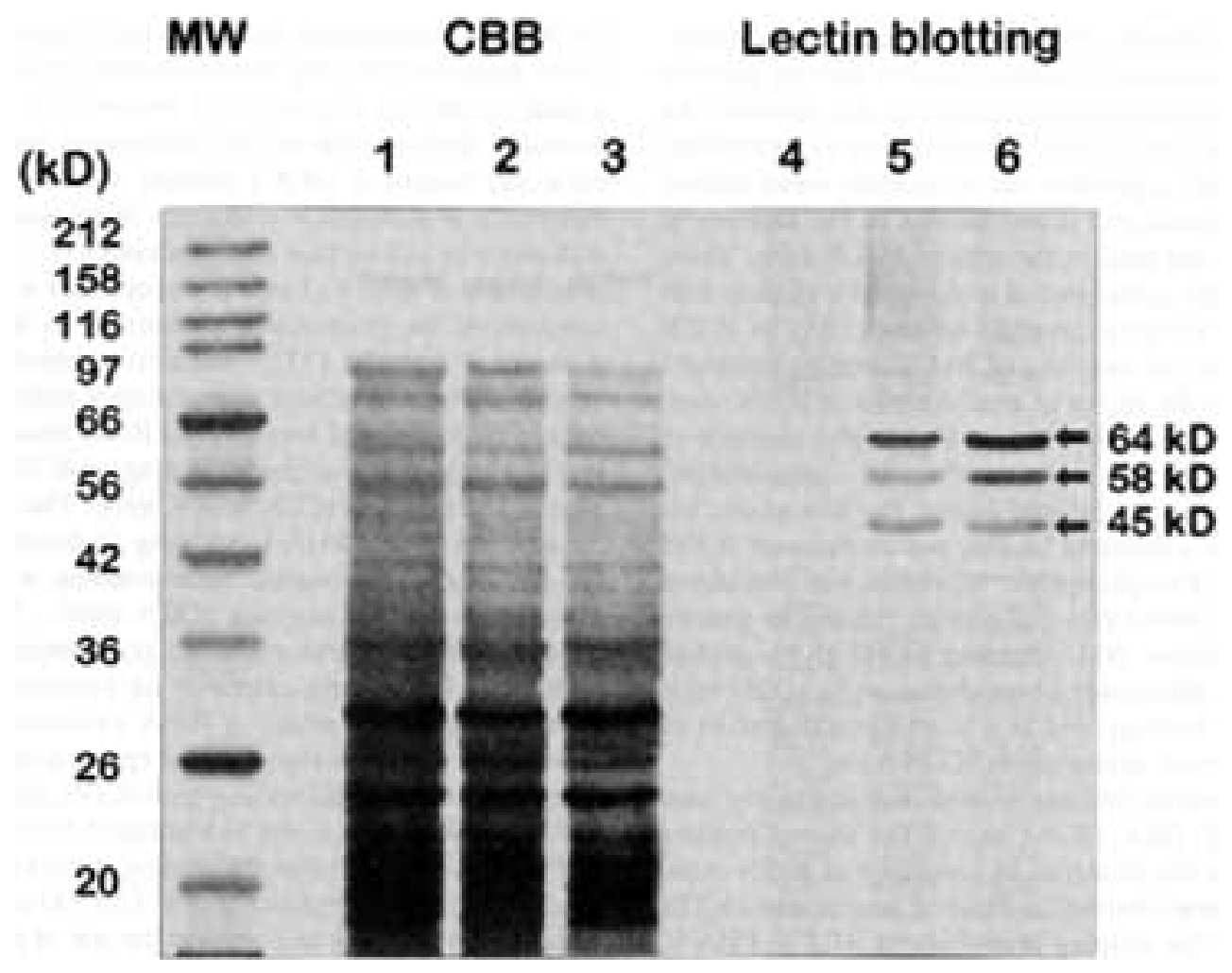

Fig. 4. Lectin blots stained with Coomassie brilliant blue ( $\mathrm{M}$ and lanes 1, 2 and 3) and Bandeiraea simplicifolia lectin-I (BSL-I) (lanes 4, 5 and 6). Lane M: molecular markers; lane 1 and 4: kidney lysate from ICR mouse; lane 2 and 5: kidney lysate from heterozygous ICGN mouse; and lane 3 and 6: kidney lysate from homozygous ICGN mouse. Characteristic glycoprotein bands with molecular weights of 45, 58 and $64 \mathrm{kD}$ (arrows) were detected in heterozygous and homozygous ICGN mice (lanes 5 and 6), but not in ICR mouse (lane 4). Increased staining of these bands was seen in homozygous ICGN mouse kidney (lane 6).

from kidney lysates of ICR, heterozygous and homozygous ICGN mice (Fig. 4; lanes 1, 2 and 3, respectively). When the separated proteins were stained with BSL-I, three distinct bands $(45,58$ and $64 \mathrm{kD})$ of ICGN mouse kidney were observed (Fig. 4; lanes 5 and 6, arrows). A strong band with a molecular mass of $64 \mathrm{kD}$ was seen in the homozygous ICGN mouse kidney. These changes in the staining intensity of kidney glycoproteins were consistent with the histochemical results described above.

\section{DISCUSSION}

Some lectins, especially BSL-I, showed characteristic staining patterns in ICGN mouse kidneys with renal failure. BSL-I showed positive staining in the glomeruli of both homozygous and heterozygous ICGN mice, but those of ICR mice were negative, and the staining intensity of BSLI in the glomeruli of homozygous ICGN mice was stronger than that of heterozygous ICGN mice. Positive staining of BSL-I in distal tubules was only observed in homozygous ICGN mice. Moreover, lectin blot analysis for BSL-I revealed characteristic glycoproteins bands with molecular weights of 45,58 and $64 \mathrm{kD}$ in heterozygous and homozygous ICGN mice but not in ICR mice, and increased staining was observed in homozygous ICGN mice. These three specific glycoproteins may be key factors in the pathogenesis of renal failure and are considered to be good markers for it. BSL-I consists of five isolectins, which are tetrameric structures composed of various combinations of two different subunits designated as $\mathrm{A}$ and $\mathrm{B}$ (BSL- $\mathrm{A}_{4}$, BSL$\mathrm{A}_{3} \mathrm{~B}_{1}, \mathrm{BSL}-\mathrm{A}_{2} \mathrm{~B}_{2}, \mathrm{BSL}-\mathrm{A}_{1} \mathrm{~B}_{3}$ and $\mathrm{BSL}-\mathrm{B}_{4}$ ) [19]. The A-subunit exhibits a primary specificity for $\alpha-D-N$ acethylgalactopyranosyl ( $\alpha$-D-GalNAcp) groups and also reacts weakly with $\alpha$-D-galactopyranosyl ( $\alpha$-D-Galp) group. Binding patterns of BSL-A 4 , homo-tetramer of A-subunits, in kidney sections were different among normal and diabetic mice, and different staining patterns have been seen among mouse strains (C57BL/6J, ICR, BALB/c and NSY) [3537]. The B-subunit shows a high degree of specificity toward $\alpha$-D-Galp but not $\alpha$-D-GalNAcp groups. BSL-B 4 , homo-tetramer of B-subunits, exhibited binding to the glomerular and peritubular basement membrane in frozen sections of mouse and rabbit kidneys, but not in those of humans [28]. Biotinylated BSL-I used in the present study is a mixture of five isolectins, binds mainly with $\alpha$-D-Galp groups[19]. Miyamoto et al. [12, 13] showed that 
histochemical staining of BSL-I demonstrated speciesspecific and strain-specific patterns, and its staining patterns specifically correspond to physiopathological condition. As described above, BSL-I specifically bound to endothelial cells of glomerular capillaries and of capillary vessel located among the proximal and distal tubules in the kidneys of ICGN mice, but not those in the kidneys of ICR mice. These histological results corresponded to the specific binding with 45, 58 and $64 \mathrm{kD}$ glycoproteins detected only in ICGN mice. Moreover, the intensity of BSL-I staining increased corresponding to the degree of renal disorder in ICGN mice, mainly reflecting the increase in $64 \mathrm{kD}$-glycoprotein in ICGN mice with severe nephrotic disorder. Interestingly, the ICGN strain was established from the ICR strain, but BSL-I exhibited a different binding pattern between ICGN and ICR mice. This phenotypic difference was considered to be based on hereditary differences related to genetic nephrotic syndrome. BSL-I binding $64 \mathrm{kD}$-glyco- protein may be one of the causes of renal disease in ICGN mice with unknown etiology and is a good clinical marker of hereditary nephrotic syndrome in ICGN mice.

Further interesting findings of lectin histochemistry were as follows: AAL, DBA, UEA-I and s-WGA showed positive staining only in the glomeruli of homozygous ICGN mice, but no staining was observed in those of heterozygous ICGN or ICR mice. The staining intensities of RCA-I, PHA-E, PHA-L, LCA and ECL in the glomeruli of homozygous ICGN mice were stronger than those of heterozygous ICGN and ICR mice. The present findings indicated that these lectins with which strong staining was demonstrated in homozygous ICGN mice with severe glomerular disorder may be good indicators for the diagnosis and prognosis of glomerular lesions. Moreover, positive staining with SSA was only observed in the proximal tubules of homozygous ICGN mice, and positive staining with BSL-I was only demonstrated in distal tubules of homozygous ICGN mice. In proximal tubules, positive staining with s-WGA was observed in both homozygous and heterozygous ICGN mice but not in ICR mice. Stronger levels of staining with DSL, AAL, PHA-E and PHA-L were demonstrated in homozygous ICGN mice compared with heterozygous ICGN and ICR mice. These lectins, whose stronger staining was seen in homozygous ICGN mice with severe renal tubular lesion, are considered to be good probes for histopathological diagnosis and prognosis of tubular disorders.

It is also interesting that the glomerular basement membrane is the obvious site with ultrastructural alterations observed as early as 5 weeks old as described in our previous report [22]. In the mesangium of ICGN mice, the histochemical intensities of RCA-I, PHA-E, PHA-L and LCA staining increased corresponding to nephritis aggravation. Moreover, no histochemical staining with AAL, DBA, UEA-I or s-WGA was observed in the mesangium of heterozygous ICGN or ICR mice, but positive staining with these lectins was demonstrated in homozygous ICGN mice. In various human renal diseases, loss of UEA-
I binding glycoconjugate was observed in the sclerotic areas of the glomeruli $[35,36]$, and this lectin is considered to be a good marker for diagnosis and prognosis of human renal diseases. Such species-specific discrepancy between ICGN mice and human in UEA-I staining may be caused by a difference in pathogenic molecules or by species-specific difference in cell-surface carbohydrates [7]. As described above, loss of BSL-A 4 binding glycoprotein accompanying sclerosis of the glomeruli was observed in diabetic mice with renal disorder [37]. Moreover, brush borders of extended proximal tubules were strongly stained with SSA only in the kidneys of homozygous ICGN mice with severe renal disorders, but no such staining with SSA was ever seen in heterozygous ICGN or ICR mice. The intensities of AAL, PHA-E and PHA-L staining in brush borders of proximal tubules increased corresponding with nephritis aggravation in homozygous ICGN mice. There was a relation between alterations in the glycoconjugates stained with these lectins and extension of proximal tubules in homozygous ICGN mice. s-WGA exhibited a granular staining pattern in proximal tubular epithelia of a portion of heterozygous and of all homozygous ICGN mice, but not in ICR mice. Recently, in situ hybridization for erythropoietin mRNA demonstrated that the cortical peritubular cells are renal erythropoietin-producing cells [11]. Erythropoietin is a circulating hormone that governs the rate of red blood cell production. We observed that all homozygous ICGN mice developed severe anemia, and that histopathological regression of cortical peritubular cells and abnormal histochemical staining with some lectins in the peritubular area. Thus, regressive changes in cortical peritubular cells, erythropoietin-producing cells, may cause severe anemia in homozygous ICGN mice. The present results suggested that some specific lectins can be used for histopathological diagnosis and prognosis of the development of renal glomerular and tubular diseases including hereditary nephrotic syndrome.

In conclusion, histopathological lectin staining can provide important information for diagnosis and prognosis of the development of hereditary nephrotic lesions. The lectins with which stronger staining was seen in homozygous ICGN mice with severe renal tubular lesions are good probes to investigate the molecular mechanism of renal disorders in ICGN mice. Particularly, three BSL-I binding glycoproteins $(45,58$ and $64 \mathrm{kD}$; especially $64 \mathrm{kD}$ glycoprotein), which may be the pathogenic factors causing renal disease in ICGN mice, are good tools to elucidate the etiology of the renal syndrome in ICGN mice.

ACKNOWLEDGEMENTS. Part of this work was supported by a Grant-in-Aid to N. M. from the Ministry of Education, Science, Sports and Culture of Japan, and by a Grant to N. M. from the Takeda Foundation and from the Itoh Memorial Foundation. We are grateful to Drs. K. Ogawa and K. Taniguchi (Iwate University, Morioka, Japan) for advice on lectin histochemistry. 


\section{REFERENCES}

1. Bradford, M. M. 1976. A rapid and sensitive method for the quantitation of microgram quantities of protein utilizing the principle of protein-dye binding. Anal. Biochem. 72: 248254.

2. Denis, J. W., Laferte, S., Waghorne, C., Breitman, M. L. and Kerbel, R. S. 1987. Beta 1-6 branching of Asn-linked oligosaccharides is directly associated with metastasis. Science 236: 582-585.

3. Hsu, S. M. and Raine, L. 1982. Versatility of biotin-labeled lectins and avidin-biotin-peroxidase complex for localization of carbohydrate in tissue sections. J. Histochem. Cytochem. 30: 157-159.

4. Kimura, Y., Manabe, N., Nishihara, S., Matsushita,H., Tajima, C., Wada, S. and Miyamoto, H. 1999. Up-regulation of the $\alpha 2,6$-sialyltransferase messenger ribonucleic acid increases glycoconjugates containing $\alpha 2,6$-linked sialic acid residues in granulosa cells during follicular atresia of porcine ovaries. Biol. Reprod. 60: 1475-1482.

5. Kimura, Y., Manabe, N., Matushita, H., Imai, Y., Myoumoto, A. and Miyamoto, H. 1998. Examination of granulosa cell glycoconjugates which change during follicular atresia in the pig ovary. J. Reprod. Dev. 44: 35-44.

6. Kuroki, T., Kubota, A., Miki, Y., Yamamura, T. and Utsunomiya, J. 1991. Lectin staining of neoplastic and normal background colorectal mucosa in nonpolyposis and polyposis patients. Dis. Colon Rectum. 34: 679-684.

7. Lis, H. and Sharon, N. 1986. Lectins as molecules and as tools. Ann. Rev. Biochem. 55: 35-67.

8. Manabe, N., Chvallier, M., Chossegros, P., Causse, X., Guerret, S., Trépo, C. and Grimaud, J-A. 1993. Interferon- $\alpha_{2 b}$ therapy reduces liver fibrosis in chronic non-A, non-B hepatitis: a quantitative histological evaluation. Hepatology 18: 1344-1349.

9. Manabe, N., Furuya, Y., Nagano, N., Yagi, M., Kuramitsu, K. and Miyamoto, H. 1995. Immunohistochemical quantitation for extracellular matrix proteins in rats with glomerulonephritis. Nephron 71: 79-86.

10. Manabe, N., Furuya, Y., Nagano, N. and Miyamoto, H. 1994. Immunohistochemical microquantitation method for type I collagen in kidney histological section of the rats. J. Vet. Med. Sci. 56: 147-150.

11. Maxwell, P. H., Ferguson, D. J. P., Nicholls, L. G., Iredale, J. P., Pugh, C. W., Johnson, M. H. and Ratcliffe, P. J. 1997. Sites of erythropoietin production. Kidney Int. 51: 393-401.

12. Miyamoto, M., Manabe, N., Kuramitsu, K., Kuribayashi, Y., Tamura, K., Furuya, Y., Nagano, N., Fukumoto, M. and Miyamoto H. 1997. Lectin histochemistry in rat liver fibrosis induced by heterologous serum sensitization. J. Vet. Med. Sci. 59: 681-687.

13. Miyamoto, M., Manabe, N., Uchio, K., Kuramitsu, K., Kuribayashi, Y., Tamura, K., Furuya, Y., Nagano, N. and Miyamoto, H. 1998. Characteristics of lectin staining patterns assessed by a modified sensitive thermo-method in rat livers with heterologous serum-induced fibrosis. J. Vet. Med. Sci. 60: 953-960.

14. Mizuno, S., Horikawa, Y., Okamoto, M. and Kurosawa, T. 1998. Preventive effect of ACE inhibitor on interstitial myofibroblast formation and matrix deposition in a nephrotic model. Renal Failure 20: 481-491.

15. Mizuno, S., Kurosawa, T., Matsumoto, K., Mizuno-Horikawa, Y., Okamoto, M. and Nakamura, T. 1998. Hepatocyte growth factor prevents renal fibrosis and dysfunction in a mouse model of chronic renal disease. J. Clin. Invest. 101: 18271834.

16. Mizuno, S., Mizuno-Horikawa, Y., Yue-Bing, F., Okamoto, M. and Kurosawa, T. 1999. Nephrotic mice (ICGN strain): A model of diffuse mesangial sclerosis in infantile nephrotic syndrome. Am. J. Nephrol. 19: 73-82.

17. Mizuno, S., Yue-Bing, F., Okamoto, M., Horikawa, Y. and Kurosawa, T. 1997. Diffuse glomerulosclerosis without tubular injury does not directly manifest renal dysfunction in nephrotic mice (ICGN strain). Exp. Nephrol. 5: 498-507.

18. Muramatsu, T. 1988. Alterations of cell-surface carbohydrates during differentiation and development. Biochimie 70: 1587 1596.

19. Murphy, L. A. and Goldstein, I. J. 1977. Five- $\alpha-$ galactopyranosyl-binding isolectins from Bandeiraea simplicifolia seeds. J. Biol. Chem. 252: 4739-4742.

20. Nakamura, T., Ebihara, I., Tomino, Y. and Koide, H. 1995. Effect of a specific endothelin A receptor antagonist on murine lupus nephritis. Kidney Int. 47: 481-489.

21. Ogura, A., Asano, T. and Fujimura, H. 1994. Animal model of human disease: inherited nephrotic syndrome. Comp. Pathol. Bull. 26: 3-6.

22. Ogura, A., Asano, T., Matsuda, J. and Fujimura, H. 1991. Evolution of glomerular lesions in nephrotic ICGN mice: serial biopsy study with electron microscopy. J. Vet. Med. Sci. 53: 513-515.

23. Ogura, A., Asano, T., Matsuda, J., Koura, M., Nakagawa, M., Kawaguchi, H. and Yamaguchi, Y. 1990. An electron microscopic study of glomerular lesion in hereditary nephrotic mice (ICGN strain). Virchows Arch. A Pathol. Anat. 417: 223-228.

24. Ogura, A., Asano, T., Matsuda, J., Takano, K., Nakagawa, M. and Fukui, M. 1989. Characteristics of mutant mice (ICGN) with spontaneous renal lesion: a new model for human nephrotic syndrome. Lab. Anim. 23: 169-174.

25. Ogura, A., Asano, T., Matsuda, J., Noguchi, Y., Yamamoto, Y., Takano, K. and Nakagawa, M. 1989. Development of nephrotic ICGN mice: the origin, reproductive ability, and incidence of glomerulonephritis. Exp. Anim. (Tokyo) 38: 349352.

26. Ogura, A., Asano, T., Suzuki, O., Yamamoto, Y., Noguchi, Y., Kawaguchi, H. and Yamaguchi, Y. 1994. Hereditary nephrotic syndrome with progression to renal failure in a mouse model (ICGN strain): clinical study. Nephron 68: 239-244.

27. Ogura, A., Fujimura, H., Asano, T., Koura, M., Naito, I. and Kobayashi, Y. 1995. Early ultrastructural glomerular alteration in neonatal nephrotic mice (ICGN strain). Vet. Pathol. 32: 321-323.

28. Peters, B. P. and Goldstei, J. 1979. The use of fluoresceinconjugated Bandeiraea simplicifolia $\mathrm{B}_{4}$-isolectin as a histochemical reagent for the detection of alpha-Dgalactopyranosyl groups: their occurrence in basement membranes. Exp. Cell Res. 120: 321-334.

29. Raiji, L. S., Azar, S. and Keane, W. 1984. Mesangial immune injury, hypertension, and progressive glomerular damage in Dahl rats. Kidney Int. 26: 137-143.

30. Shih, W., Hinbes, W. H. and Neilson, E. G. 1988. Effects of cyclosporin A on the development of immune-mediated interstitial nephritis. Kidney Int. 33: 1113-1118.

31. Tatsumi, H., Satoh, S., Okamoto, M., Nakamura, M., Asano, T. and Kurosawa, T. 1995. Morphological studies on the kidney of the spontaneous nephrotic (ICGN) mice in the late stage. Acta Anat. Nippon. 70: 96-106. 
32. Uchio, K., Manabe, N., Kinoshita, A., Tamura, K., Miyamoto, M., Ogura, A., Yamamoto, Y. and Miyamoto, H. 1999. Abnormalities of extracellular matrices and transforming growth factor $B 1$ localization in the kidney of the hereditary nephrotic mice (ICGN Strain). J. Vet. Med. Sci. 61: 769-776.

33. Uchio, K., Manabe, N., Tamura, K., Miyamoto, M., Ogura, A., Yamamoto, Y. and Miyamoto, H. Decreased activities of matrix metalloproteinases in the kidneys of hereditary nephrotic mice (ICGN Strain). Nephron (in press).

34. Yamamoto, K., Makino, Y., Yoshioka, T., Kobayashi, H., Tomita, M. and Tsuji, T. 1992. Quantitative analysis of activated Kupffer cells in viral hepatitis: application of computer image analysis for lectin histochemistry. Liver 12: 199-204.

35. Yonezawa, S., Irisa, S., Nakamura, T., Uemura, S., Otuji, Y.,
Ohi, Y. and Sato, E. 1983. Deposition of $\alpha 1$-antitrypsin and loss of glycoconjugate carrying Ulex europaeus agglitinin-I binding sites in glomerular sclerotic process: phenomena common to chronic pyelonephritis and chronic diffuse proliferative glomerulonephritis. Nephron 33: 38-43.

36. Yonezawa, S., Nakamura, T., Irisa, S., Otuji, Y. and Sato, E. 1985. Ulex europaeus agglitinin I staining of human glomerular lesions using a highly sensitive immunoperoxidase method in paraffin sections. Nephron 35: 187-189.

37. Yonezawa, S., Shibata, M., Shimizu, T., Nakamura, T. and Sato, E. 1986. Glifffonia simplicifolia $\mathrm{I}_{-} \mathrm{A}_{4}$ staining of mice glomerular tufts and its alteration in diabetic mice. Acta Pathol. Jpn. 36: 1653-1658. 